\title{
A Distributed Consensus-Based Cooperative Spectrum-Sensing Scheme in Cognitive Radios
}

\author{
Zhiqiang Li, F. Richard Yu, Senior Member, IEEE, and Minyi Huang, Member, IEEE
}

\begin{abstract}
In cognitive radio (CR) networks, secondary users can cooperatively sense the spectrum to detect the presence of primary users. In this paper, we propose a fully distributed and scalable cooperative spectrum-sensing scheme based on recent advances in consensus algorithms. In the proposed scheme, the secondary users can maintain coordination based on only local information exchange without a centralized common receiver. Unlike most of the existing decision rules, such as the oR-rule or the 1-out-of-N rule, we use the consensus of secondary users to make the final decision. Simulation results show that the proposed consensus scheme can have significant lower missing detection probabilities and false alarm probabilities in CR networks. It is also demonstrated that the proposed scheme not only has proven sensitivity in detecting the primary user's presence but also has robustness in choosing a desirable decision threshold.
\end{abstract}

Index Terms-Cognitive radios (CRs), consensus, cooperative spectrum sensing, random graphs.

\section{INTRODUCTION}

C OGNITIVE radio (CR), which has been introduced in [1], is an enabling technology that allows unlicensed (secondary) users to operate in licensed spectrum bands. This can help to overcome the lack of available spectrum in wireless communications. Since CRs are considered to be lower priority and are secondary users of the spectrum allocated to a primary user, a fundamental requirement is to avoid the interference to potential primary users in their vicinity [2]. In addition, primary user networks have no requirement to change their infrastructure for spectrum sharing with CRs. Therefore, secondary users should be able to independently detect the presence of primary users through continuous spectrum sensing. Such spectrum sensing can be conducted either noncooperatively (individually), in which each secondary user conducts radio detection and makes a decision by itself, or cooperatively, in which a group of secondary users perform spectrum sensing by collaboration.

Manuscript received November 7, 2008; revised April 16, 2009, June 19, 2009, and July 20, 2009. First published September 1, 2009; current version published January 20, 2010. This work was supported in part by the Natural Sciences and Engineering Research Council of Canada. The review of this paper was coordinated by Dr. Y.-C. Liang.

Z. Li and F. R. Yu are with the Department of Systems and Computer Engineering, Carleton University, Ottawa, ON K1S 5B6, Canada (e-mail: zlia@sce.carleton.ca; richard_yu@carleton.ca).

M. Huang is with the School of Mathematics and Statistics, Carleton University, Ottawa, ON K1S 5B6, Canada (e-mail: mhuang@math.carleton.ca).

Color versions of one or more of the figures in this paper are available online at http://ieeexplore.ieee.org.

Digital Object Identifier 10.1109/TVT.2009.2031181
Cooperative spectrum sensing has recently been studied. There are several advantages offered by cooperative spectrum sensing over noncooperative spectrum sensing [3]-[12]. If a secondary user is in the condition of deep shadowing and fading, it is very difficult for a secondary user to distinguish a white space from a deep shadowing effect. Therefore, a noncooperative spectrum sensing algorithm may not work well in this case, and a cooperative scheme can solve the problem by sharing the spectrum-sensing information among secondary users. Moreover, because of the hidden terminal problem, it is very challenging for single-CR sensitivity to outperform the primary user receiver by a large margin to detect the presence of primary users. For this reason, if secondary users spread out in the spatial distance and any one of them detects the presence of primary users, then the whole group can gain benefit by collaboration.

Ghasemi and Sousa [3] quantified the performance of spectrum sensing in fading environments and studied the effect of cooperation. The simulation results in [3] indicate that significant performance enhancements can be achieved through cooperation. Ganesan and Li [4] studied the possibility of forwarding the signal with higher SNR to the one on the boundary of the decidability region of the primary user. The performance is evaluated under correlated shadowing and user compromise in [5]. When the exchange of observations from all secondary users to the common receiver is not applicable, Peh and Liang [6] showed that it is still worth doing through cooperation with a certain number of users with relatively higher SNR. Moreover, in [8], a linear-quadratic fusion strategy is designed with the consideration of the correlation between the nodes. To further reduce the computational complexity, Quan et al. [9] proposed a heuristic approach to develop an optimal linear framework during cooperation. Sensing-throughput tradeoff is analyzed in [10] for both multiple minislots and multiple secondary-user cooperative sensing.

Although some research activities have been conducted in cooperative spectrum sensing, most of them use a common receiver (fusion center) to perform data fusion for the final decision, regardless of whether the primary user is present. However, a common receiver may not be available in some CR-based networks, such as mobile ad hoc networks. Moreover, as indicated in [5], gathering the entire received data in one place may be very difficult under practical communication constraints. In addition, Sun et al. [13] studied the reporting channels between the cognitive users and the common receiver. The results showed that there are limitations in the performance of cooperation when the reporting channels to the common receiver are under deep fading. 
In this paper, we present a spectrum-sensing consensus scheme without using a common receiver. Our scheme is based on recent advances in consensus algorithms [14]. An important motivational background of this area is initially related to the study of complex natural phenomena, including flocking of birds, schooling of fish, swarming of honeybees, among others [15]. A key feature of such models is that the population of agents can maintain coordination based on local interactions without the centralized information exchange. Recently, consensus problems have also played a crucial role in spacial distributed control models [14], wireless sensor networks [16], and multiagent coordination over noisy networks [17]. Concerning our current spectrum-sensing models, the basic requirement is for the secondary users to collectively determine the presence of the primary user, which can be viewed as a typical multiagentcoordination situation. We especially intend to develop consensus approaches for efficient spectrum sensing. There are four distinct features in the proposed scheme.

1) It is a fully distributed and scalable scheme. Unlike existing schemes, there is no need for a common receiver to perform data fusion for the final decision. A secondary user only needs to set up a neighborhood with users having the desired channel characteristics, such as lineof-sight (LOS) users or even probabilistic link failures.

2) Unlike most decision rules, such as the OR-rule or the 1 -out-of-N rule, which were adopted in the existing schemes, we use the consensus of secondary users to make the final decision. Therefore, the proposed scheme can leverage the detection results among users in severe wireless fading networks.

3) The proposed spectrum-sensing scheme uses a consensus algorithm to cope with two underlying network models, i.e., one with fixed bidirectional graphs and one with random graphs.

4) Since the CR paradigm imposes humanlike characteristics (e.g., learning, adaptation, and cooperation) in wireless networks, the bio-inspired consensus algorithm used in this paper can provide some insight into the design of future CR networks.

Extensive simulation results illustrate the effectiveness of the proposed scheme. It is shown that the proposed scheme can have both lower missing detection probability and lower false alarm probability, compared with existing schemes. In addition, it is able to make better detection when secondary users undergo worse fading (lower average SNR). Last, but not least, with the help of this scheme, a fixed threshold is feasible, which can take active effect in different fading channels.

Our consensus-based approach is different from those used in distributed/decentralized detection problems [18]-[21]. In a typical distributed detection problem [18]-[20], each sensor individually forms its own discrete messages based on its local measurement and then reports to a fusion center, and there is, in general, no direct communication among the sensors. In certain models [21], a sensor may indirectly obtain information about other sensors, but this is achieved by feedback from a common fusion center.
The rest of this paper is organized as follows: Section II presents the spectrum-sensing models and underlying network models, together with the formal definition of the spectrumsensing consensus scheme. In Section III, the consensus algorithm is presented under the condition that the wireless links among secondary users are all LOS links with duplex modes. Section IV extends this consensus algorithm to take consideration of occasionally disconnected links due to channel fading and transmission errors. Some simulation results are given in Section V. Finally, we conclude this study in Section VI.

\section{SPectrum SENSING AND SECONDARY-USER NETWORK MODELING}

There are two stages in the proposed CR consensus scheme. In the first stage, secondary users use a spectrum-sensing model to make measurements about primary users at the beginning of detection. We denote the local measurement of user $i$ as $Y_{i}$. In the second stage, secondary users establish communication links with their own neighbors to locally exchange information among them and then calculate the obtained data to make a local decision, regardless of whether primary users are around. The aforementioned process in the second stage is iteratively done. At the initial time instant $k=0$, each user $i$ sets $x_{i}(0)=Y_{i}$ as the initial value of the local state variable. Next, at time $k=0,1,2, \ldots$, according to the real-time network topology (or local wireless neighborhood), users mutually transmit and receive their states and then use local computation rules to generate updated states $x_{i}(k+1)$. Those iterations are repeatedly done until all the individual states $x_{i}(k)$ converge toward a common value $x^{*}$.

Before we introduce the detailed algorithms used in our consensus scheme, the common spectrum-sensing model used in the first stage and the network model used in the second stage are to be presented, followed by the formal definition of the spectrum-sensing consensus scheme.

\section{A. Spectrum-Sensing Model}

In the first stage, secondary users make measurements about primary users at the beginning of each time slot. Three kinds of methods are widely used for spectrum sensing [22]. A matched filter is theoretically optimal, but it needs prior knowledge of the primary system, which means higher complexity and cost in developing adaptive sensing circuits for different primary wireless systems. Energy detection is suboptimal, but it is simple to implement and does not have too much of a requirement on the position of primary users. Cyclostationary feature detection can detect the signals with very low SNR, but it still requires some prior knowledge of the primary user [13].

In this paper, we consider the modeling scenario where prior knowledge of the primary user is unknown. For implementation simplicity, an energy-detection spectrum-sensing method [3] is used. Fig. 1 shows the block diagram of an energy detector. The input band pass filter selects the center frequency $f_{s}$ and the bandwidth of interest $W$. This filter is followed by a squaring device and, subsequently, an integrator over a period of $T$. The output $Y$ of the integrator is the received energy at the 


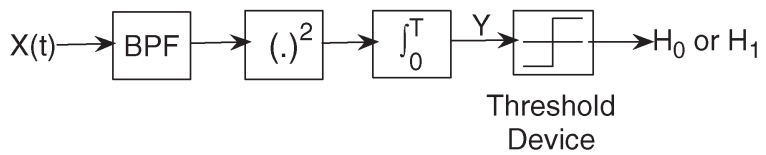

Fig. 1. Block diagram of an energy detector.

secondary user, and its distribution depends on whether the primary user signal is present or not. The goal of spectrum sensing is to decide between the following two hypotheses:

$$
x(t)= \begin{cases}n(t), & H_{0} \\ h \cdot s(t)+n(t), & H_{1}\end{cases}
$$

where $x(t)$ is the signal received by the secondary user, $s(t)$ is the primary user's transmitted signal, $n(t)$ is the additive white Gaussian noise, and $h$ is the amplitude gain of the channel. We also denote the SNR as $\gamma$. The output of integrator in Fig. 1 is $Y$, which serves as the decision statistic. Following the work of [23], $Y$ has the following form:

$$
Y= \begin{cases}\chi_{2 T W}^{2}, & H_{0} \\ \chi_{2 T W}^{2}(2 \gamma), & H_{1}\end{cases}
$$

where $\chi_{2 T W}^{2}$ and $\chi_{2 T W}^{2}(2 \gamma)$ denote random quantities with central and noncentral chi-square distributions, respectively, each with $2 T W$ degrees of freedom and a noncentrality parameter of $2 \gamma$ for the latter distribution. For simplicity, we assume that the time-bandwidth product $T W$ is an integer number, which is denoted by $m$.

Under Rayleigh fading, the gain $h$ is random, and the resulting SNR $\gamma$ would have an exponential distribution; therefore, in this case, the distribution of the output energy depends on the average SNR $(\bar{\gamma})$. When the primary user is absent, $Y$ is still distributed according to $\chi_{2 T W}^{2}$. When the primary user is present, $Y$ may be denoted as the sum of two independent random variables [24], [25], i.e.,

$$
Y=Y_{\chi}+Y_{e}, \quad H_{1}
$$

where the distribution of $Y_{\chi}$ is $\chi_{2 T W-2}^{2}$, and $Y_{e}$ has an exponential distribution with parameter $2(\bar{\gamma}+1)$.

As a summary, after $T$ seconds, each secondary user $i$ detects the energy and gets the measurement $Y_{i} \in \mathbb{R}^{+}$.

\section{B. Network Model and Consensus Notions}

In the second stage, secondary users establish communication links with their neighbors to locally exchange information among them. In our scheme, the network formed by the secondary users can be described by a standard graph model. For simplicity, this can be represented by an undirected graph (to be simply called a graph) $G=(\mathcal{N}, \mathcal{E})$ [26] consisting of a set of nodes $\{i=1,2, \ldots, n\}$ and a set of edges $\mathcal{E} \subset N \times N$. Denote each edge as an unordered pair $(i, j)$. Thus, if two secondary users are connected by an edge, it means that they can mutually exchange information. A path in $\boldsymbol{G}$ consists of a sequence of nodes $i_{1}, i_{2}, \ldots, i_{l}, l \geq 2$ such that $\left(i_{m}, i_{m+1}\right) \in \mathcal{E}$ for all $1 \leq m \leq l-1$. The graph $G$ is connected if any two distinct nodes in $G$ are connected by a path. For convenience of exposition, we often refer to node $i$ as secondary user $i$. The two names, i.e., secondary user and node, will be used interchangeably. The secondary user $j$ (node $j$ ) is a neighbor of user $i$ (node $i$ ) if $(j, i) \in \mathcal{E}$, where $j \neq i$. Denote the neighbors of node $i$ as $\mathcal{N}_{i}=\{j \mid(j, i) \in \mathcal{E}\} \subset \mathcal{N}$. The number of elements in $\mathcal{N}_{i}$ is denoted by $\left|\mathcal{N}_{i}\right|$ and called the degree of node $i$.

The Laplacian of the graph $\boldsymbol{G}$ is defined as $\boldsymbol{L}=\left(l_{i j}\right)_{n \times n}$, where

$$
l_{i j}= \begin{cases}\left|\mathcal{N}_{i}\right|, & \text { if } j=I \\ -1, & \text { if } j \in \mathcal{N}_{i} \\ 0, & \text { otherwise. }\end{cases}
$$

The matrix $\boldsymbol{L}$ defined by (4) is positive semidefinite. Furthermore, if $\boldsymbol{G}$ is a connected undirected $\operatorname{graph}$, then $\operatorname{rank}(\boldsymbol{G})=$ $n-1$ (see, e.g., [15]).

Since the cooperative spectrum-sensing problem is viewed as a consensus problem where the users locally exchange information regarding their individual detection outcomes before reaching an agreement, we give the formal mathematical definition of consensus as follows:

For the $n$ secondary users distributed according to the graph model $\boldsymbol{G}$, we assign them a set of state variables $x_{i}, i \in \mathcal{N}$. Each $x_{i}$ will be called a consensus variable, and in the cooperative spectrum-sensing context, it is essentially used by node $i$ for its estimate of the energy detection. By reaching consensus, we mean that the individual states $x_{i}$ asymptotically converge to a common value $x^{*}$, i.e.,

$$
x_{i}(k) \rightarrow x^{*} \quad \text { as } k \rightarrow \infty
$$

for each $i \in \mathcal{N}$, where $k$ is the discrete time, $k=0,1,2, \ldots$, and $x_{i}(k)$ is updated based on the previous states of node $i$ and its neighbors.

The special cases with $x^{*}=\operatorname{Ave}(x)=(1 / n) \sum_{i=1}^{n} x_{i}(0)$, $x^{*}=\max _{i=1}^{n} x_{i}(0)$, and $x^{*}=\min _{i=1}^{n} x_{i}(0)$ are called averageconsensus, max-consensus, and min-consensus, respectively. It is worth mentioning that the existing spectrum sensing algorithm with the OR-rule can be viewed as a form of max-consensus. This paper is intended to propose a cooperative spectrum-sensing scheme in the framework of averageconsensus.

\section{Spectrum Sensing With Fixed NeTwORK TOPOLOGIES}

In this section, let us assume that the secondary users have established duplex wireless connections with their desired neighbors and that the connections remain working until the consensus is reached. This kind of topology is called a fixed graph. Based on this assumption, we are going to propose the spectrum-sensing consensus algorithm.

\section{A. Consensus Algorithm}

We denote, for user $i$, its measurement $Y_{i}$ at time $k=0$ by $x_{i}(0)=Y_{i} \in \mathbb{R}^{+}$. The state update of the consensus variable for each secondary user occurs at discrete time $k=0,1,2, \ldots$, 
which is associated with a given sampling period. From $k=$ $0,1,2, \ldots$, the iterative form of the consensus algorithm can be stated as follows [15]:

$$
x_{i}(k+1)=x_{i}(k)+\epsilon \sum_{j \in \mathcal{N}_{i}}\left(x_{j}(k)-x_{i}(k)\right)
$$

where

$$
0<\epsilon<\left(\max _{i}\left|\mathcal{N}_{i}\right|\right)^{-1} \triangleq 1 / \Delta .
$$

The number $\Delta$ is called the maximum degree of the network.

This algorithm can be written in vector form

$$
\boldsymbol{x}(k+1)=\boldsymbol{P} \boldsymbol{x}(k)
$$

where $\boldsymbol{P}=\boldsymbol{I}-\epsilon \boldsymbol{L}$. Notice that the upper bound in (7) for $\epsilon$ ensures that $\boldsymbol{P}$ is a stochastic matrix, and in fact, one can further show that $\boldsymbol{P}$ is ergodic when $\boldsymbol{G}$ is connected. ${ }^{1}$ Since $\boldsymbol{G}$ is an undirected graph, all row sums and column sums of $\boldsymbol{L}$ are equal to zero. Hence, $\boldsymbol{P}$ is a doubly stochastic matrix (i.e., $\boldsymbol{P}$ is a nonnegative matrix and all of its row sums and column sums are equal to one).

We also point out that (8) uses only a particular construction of the coefficient matrix for the consensus algorithm, which is based on the graph Laplacian $\boldsymbol{L}$. As long as each node has prior knowledge of an upper bound of the maximum degree $\Delta$ of the network, the iteration may be implemented, and there is no necessity for neighboring nodes to exchange information regarding the network structure. In addition, it is possible to construct $\boldsymbol{P}$ in other forms. An alternative choice of $\boldsymbol{P}$ may be based on the so-called Metropolis weights [16] by taking

$$
\tilde{p}_{i j}= \begin{cases}\frac{1}{1+\max \left\{d_{i}, d_{j}\right\}}, & \text { if }(j, i) \in \mathcal{E} \\ 1-\sum_{j \in \mathcal{N}_{i}} \tilde{p}_{i j}, & \text { if } i=j \\ 0, & \text { otherwise }\end{cases}
$$

where $d_{i}=\left|\mathcal{N}_{i}\right|$ is the degree of node $i$. If $\boldsymbol{G}$ is a connected graph and we define $\widetilde{\boldsymbol{P}}=\left(\tilde{p}_{i j}\right)_{n \times n}$, then $\widetilde{\boldsymbol{P}}$ is an ergodic doubly stochastic matrix. When $\widetilde{\boldsymbol{P}}$ is used in (8) in place of $\boldsymbol{P}$, the state average will still be preserved as an invariant during the iterations, and our convergence analysis here is still valid. Notice that, when $\widetilde{\boldsymbol{P}}$ is used in the consensus algorithm, it is only required that any two neighboring nodes report to each other their degrees, and knowledge of the maximum degree of the network is no longer needed.

We cite a theorem concerning the convergence property of the consensus algorithm.

Theorem 1 (see, e.g., [15]): Consider a network of secondary users

$$
x_{i}(k+1)=x_{i}(k)+u_{i}(k)
$$

with topology $G$ applying the distributed consensus algorithm (6), where $u_{i}(k)=\epsilon \sum_{j \in \mathcal{N}_{i}}\left(x_{j}(k)-x_{i}(k)\right), 0<\epsilon<$

\footnotetext{
${ }^{1}$ For some network topologies, it is possible to have an ergodic matrix $P=$ $I-\epsilon L$ when $\epsilon=1 / \Delta$. For instance, if $\epsilon$ is taken as $1 / \Delta$ and, meanwhile, it is ensured that $P$ has at least one positive diagonal entry, then it can be shown that $P$ is an ergodic stochastic matrix.
}

$1 / \Delta$, and $\Delta$ is the maximum degree of the network. Let $G$ be a connected undirected graph. Then, two conditions are observed.

1) A consensus is asymptotically reached for all initial states.

2) $\boldsymbol{P}$ is doubly stochastic, and an average-consensus is asymptotically reached with the limit $x^{*}=$ $(1 / n) \sum_{i=1}^{n} x_{i}(0)$ for the individual states.

According to Theorem 1, if we choose $\epsilon$ such that $0<$ $\epsilon<1 / \Delta$, then an average consensus is ensured, and the final common value $x^{*}=(1 / n) \sum_{i=1}^{n} x_{i}(0)$ will be the average of the initial vector $\boldsymbol{x}(\mathbf{0})$ or, equivalently, the average of $\boldsymbol{Y}^{\boldsymbol{T}}=$ $\left\{Y_{1}, Y_{2}, \ldots, Y_{n}\right\}$, which has been obtained during the energydetection stage.

Finally, by comparing the average consensus result $x^{*}$ with a predefined threshold $\lambda$ based on Fig. 1, every secondary user $i$ locally gets the final data fusion, i.e.,

$$
\text { Decision } \boldsymbol{H}= \begin{cases}1, & x^{*}>\lambda \\ 0, & \text { otherwise. }\end{cases}
$$

\section{B. Performance of the Consensus Algorithm}

It is quite apparent that the convergence rate is yet another interesting issue in evaluating the performance of the spectrumsensing consensus algorithm. This is due to the fact that secondary users must continuously detect the presence of primary users and back up as soon as possible on recognizing such incident. From this point of view, the speed of reaching a consensus is the key in the design of the network topology and the analysis of the performance of a consensus algorithm for a given spectrum-sensing network. For the connected undirected graph $G$, the aforementioned algorithm can ensure exponential convergence rate, where the error can be parameterized in the form $O\left(e^{-\delta t}\right)$ with the exponent $\delta>0$. To have some bound estimate for the parameter $\delta$, we first recall that $\boldsymbol{P}=\boldsymbol{I}-\epsilon \boldsymbol{L}$. Since $L$ is a positive semidefinite matrix, denote its $n$ eigenvalues by

$$
0=\lambda_{1}<\lambda_{2} \leq \cdots \leq \lambda_{n} .
$$

Here, $\lambda_{2}>0$ since the undirected graph $\mathbf{G}$ is connected, which ensures that the rank of $\boldsymbol{L}$ is equal to $n-1$ [27]. The second smallest eigenvalue $\lambda_{2}$ of $\boldsymbol{L}$ is usually called the algebraic connectivity of the undirected graph $G$. Then, the second largest absolute value of the eigenvalues of $\boldsymbol{P}$ is determined as $\alpha(\epsilon)=\max \left\{\left|1-\epsilon \lambda_{2}\right|,\left|1-\epsilon \lambda_{n}\right|\right\}$, which can be verified to satisfy $\alpha(\epsilon)<1$. By using standard results in nonnegative matrix theory (see, e.g., [28]), we can obtain an upper bound for $\delta$. In fact, we can take $\delta$ as any value in the interval $(0,-\ln \alpha(\epsilon))$. We also remark that similar convergence rate estimates can be carried out when general weight matrices in averaging are used.

Since $\boldsymbol{P}$ has a unit eigenvalue, we see that the difference between the first two largest absolute values of the eigenvalues of $\boldsymbol{P}$ is given as $g(\epsilon)=1-\alpha(\epsilon)$, which is customarily called the spectral gap of $\boldsymbol{P}$. In general, the greater the $g(\epsilon)$, the greater the upper bound $-\ln \alpha(\epsilon)$ for the exponent $\delta$ and the faster the convergence of the consensus algorithm. In practical implementations, it is desirable to choose a suitable value for 
$\epsilon$ to increase the spectral gap $g(\epsilon)$, whereas $\boldsymbol{P}$ is ensured to be ergodic.

\section{Spectrum Sensing With Random NETWORK CONNECTIVITY}

In Section III, it has been assumed that any two neighboring nodes can reliably exchange data at all times. Hence, the network topology remains unchanged during the overall time period of interest. This kind of network modeling may not be accurate in certain situations. For example, fading of wireless signals can cause packet errors, which will result in wireless link failures for that period. Furthermore, even under LOS channels, moving objects between neighboring nodes may temporarily affect signal reception. For the aforementioned reasons, in this section, we consider a more realistic internode communication model with random link failures. Unlike the previous model, which is based on fixed bidirectional graphs, the new model is based on random graphs. Nevertheless, similar to the previous fixed topology scenario, for the random-graphbased modeling given here, we still consider bidirectional links when two nodes can communicate.

\section{A. Random Graph Modeling of the Network Topology}

Before characterizing the random connectivity of the network of all secondary users, let us first introduce a fixed undirected graph $\boldsymbol{G}=(\mathcal{N}, \mathcal{E})$, which describes the maximal set of communication links when there is no link failure. Due to the random link failures, at time $k$, the interuser communication is described by a subgraph of $\boldsymbol{G}$ denoted by $\boldsymbol{G}(k)=(\mathcal{N}, \mathcal{E}(k))$, where $\mathcal{E}(k) \subset \mathcal{E}$; the edge $(j, i) \in \mathcal{E}(k)$ if and only if nodes $j$ and $i$ can communicate at time $k$, where $(j, i) \in \mathcal{E}$. Thus, the (undirected) graph $\boldsymbol{G}(k)$ is generated as the outcome of random link failures. Note that an edge $(j, i)$ never appears in $\boldsymbol{G}(k)$ if it is not an edge of $\boldsymbol{G}$. The neighbor set of node $i$ is $\mathcal{N}_{i}(k)=\{j \mid(j, i) \in \mathcal{E}(k)\}$ at time $k$. The number of elements in $\mathcal{N}_{i}(k)$ is denoted by $\left|\mathcal{N}_{i}(k)\right|$. At time $k \geq 0$, the adjacency matrix of $\boldsymbol{G}(k)$ is defined as $\boldsymbol{A}(k)=\left(\alpha_{j i}(k)\right)_{1<j, i<|\mathcal{N}|}$, where $\alpha_{j i}(k)=1$ if $(j, i) \in \mathcal{E}(k)$ and $\alpha_{j i}(k)=0$ otherwise. It is clear that the graph $\boldsymbol{G}(k)$ is completely characterized by the random matrix $\boldsymbol{A}(k)$.

Concerning the statistical properties of link failures, we assume that all links (each associated with an edge in the graph $\boldsymbol{G}$ ) independently fail with the same probability $p \in$ $(0,1)$. For notational simplicity, we use the same parameter $p$ to model the failure probability. The generalization of the modeling and analysis to link-dependent failure probabilities is straightforward.

\section{B. Algorithm With Random Graphs}

For the random link failure-prone model, the two spectrumsensing stages introduced in Section II are still applicable. In the first stage, each node performs the radio detection and computes the measurements according to (1). During the second stage, at time $k$, each node exchanges state information with its neighbors and performs the corresponding computation to generate its state update $x_{i}(k+1)$. Let $\Delta$ be the maximum degree of the graph $\boldsymbol{G}$, and take $\epsilon \in(0,1 / \Delta)$.
The state of user $i \in \mathcal{N}$ is updated by the rule

$$
x_{i}(k+1)=x_{i}(k)+\epsilon \sum_{j \in \mathcal{N}_{i}(k)}\left[x_{j}(k)-x_{i}(k)\right]
$$

where $\epsilon$ is a predetermined constant step size. If $\mathcal{N}_{i}(k)=\emptyset$ (empty set), (12) reduces to $x_{i}(k+1)=x_{i}(k)$.

Theorem 2: Under the independent link failure assumption, the algorithm (12) ensures average consensus, i.e., $\lim _{k \rightarrow \infty} x_{i}(k)=(1 / n) \sum_{j=1}^{n} x_{j}(0)$ for all $i \in \mathcal{N}$, with probability one. If, in addition, $E|x(0)|^{2}<\infty$ and $x(0)$ is independent of the sequence of adjacency matrices $\mathbf{A}(k), k=0,1, \ldots$, then each $x_{i}(k)$ converges to $(1 / n) \sum_{j=1}^{n} x_{j}(0)$ in mean square with an exponential convergence rate.

Proof: We can write algorithm (12) in vector form

$$
\boldsymbol{x}(k+1)=[\boldsymbol{I}-\epsilon \boldsymbol{L}(k)] \boldsymbol{x}(k)
$$

where $\boldsymbol{L}(k)$ is the Laplacian of the graph $\boldsymbol{G}(k)$. For a vector $\boldsymbol{z}=\left(z_{1}, \ldots, z_{n}\right)^{T}$, denote the Euclidean norm $|\boldsymbol{z}|=$ $\left(\sum_{i=1}^{n} z_{i}^{2}\right)^{1 / 2}$. For any given sample point, we can show that $\boldsymbol{M}(k)=\boldsymbol{I}-\epsilon \boldsymbol{L}(k)$ is a symmetric aperiodic stochastic matrix so that it has all its eigenvalues within the interval $(-1,1]$ (see, e.g., [28]); therefore, $\boldsymbol{M}(k)$ determines a paracontracting map [16], [29] in the sense $\boldsymbol{M}(k) \boldsymbol{z} \neq \boldsymbol{z}$ if and only if $|\boldsymbol{M}(k) \boldsymbol{z}|<$ $|\boldsymbol{z}|$. For $\boldsymbol{M}(k)$, we denote its fixed-point subspace $\mathcal{H}(\boldsymbol{M}(k))=$ $\left\{\boldsymbol{z} \in \mathbb{R}^{n} \mid \boldsymbol{M}(k) \boldsymbol{z}=\boldsymbol{z}\right\}$.

By the assumption on the independent link failures, we see that, with probability one, $\boldsymbol{G}(k)=\boldsymbol{G}$ for an infinite number of times $k$. Let $\Omega$ denote the underlying probability sample space. Thus, after excluding a set $A_{0}$ of zero probability, for all $\omega \in \Omega \backslash A_{0}, \boldsymbol{G}(k)=\boldsymbol{G}$ infinitely often, with the associated Laplacian being $\boldsymbol{L}(k)=\boldsymbol{L}$. Hence, for each $\omega \in \Omega \backslash A_{0}$, $x(k)$ converges to a point in the space $\mathcal{H}(\boldsymbol{I}-\epsilon \boldsymbol{L})=\{z \in$ $\left.\mathbb{R}^{n} \mid \boldsymbol{L} z=0\right\}$ when $k \rightarrow \infty$. Furthermore, $\left\{\boldsymbol{z} \in \mathbb{R}^{n} \mid \boldsymbol{L} \boldsymbol{z}=\right.$ $0\}=\operatorname{span}\left\{1_{n}\right\}$ since $G$ is a connected undirected graph.

On the other hand, it is straightforward to check that $(1 / n) \sum_{j=1}^{n} x_{j}(k)$ remains as a constant since $\boldsymbol{M}(k)$ is a doubly stochastic matrix (i.e., nonnegative matrix with all row sums and column sums equal to one). Now it follows that each $x_{i}(k)$ converges to $(1 / n) \sum_{j=1}^{n} x_{j}(0)$ with probability one, as $k \rightarrow \infty$.

We continue to analyze mean square convergence. Since $E|\boldsymbol{x}(0)|^{2}<\infty$ and $\sup _{i \in \mathcal{N}, k>0}\left|x_{i}(k)\right| \leq \max _{i \in \mathcal{N}}\left|x_{i}(0)\right| \leq$ $|\boldsymbol{x}(0)|$, by the probability-one convergence of $x_{i}(k)$, it follows from dominated convergence results in probability theory that $x_{i}(k)$ also converges to $(1 / n) \sum_{j=1}^{n} x_{j}(0)$ in mean square.

Now, we proceed to give an estimation of the mean square convergence rate within the random network model. Denote $\operatorname{Ave}(\boldsymbol{x}(0))=(1 / n) \sum_{j=1}^{n} x_{j}(0)$. It is straightforward to show that

$$
\begin{aligned}
& \boldsymbol{x}(k+1)-\operatorname{Ave}(\boldsymbol{x}(0)) \mathbf{1}_{n} \\
& \quad=\left[\boldsymbol{I}-(1 / n) \mathbf{1}_{n} \mathbf{1}_{n}^{T}\right][\boldsymbol{I}-\epsilon \boldsymbol{L}(k)]\left[\boldsymbol{x}(k)-\operatorname{Ave}(\boldsymbol{x}(0)) \mathbf{1}_{n}\right] \\
& \quad \equiv \boldsymbol{B}(k)\left[\boldsymbol{x}(k)-\operatorname{Ave}(\boldsymbol{x}(0)) \mathbf{1}_{n}\right] .
\end{aligned}
$$

In fact, for each $\omega \in \Omega$, by the eigenvalue distribution of the matrices $(1 / n) \mathbf{1}_{n} \mathbf{1}_{n}^{T}$ and $\boldsymbol{L}(k)$, we can show that $\boldsymbol{B}^{T}(k) \boldsymbol{B}(k)$ and, subsequently, that $E\left[\boldsymbol{B}^{T}(k) \boldsymbol{B}(k)\right]$ have $n$ real eigenvalues 
on the interval $[0,1]$. We use a contradiction argument to show that the largest eigenvalue $\rho$ of $E\left[\boldsymbol{B}^{T}(k) \boldsymbol{B}(k)\right]$ is less than one. Suppose that $\rho=1$ for $E\left[\boldsymbol{B}^{T}(k) \boldsymbol{B}(k)\right]$; then, there exists a real-valued vector $\boldsymbol{x} \neq 0$ such that

$$
\boldsymbol{x}^{T} E\left[\boldsymbol{B}^{T}(k) \boldsymbol{B}(k)\right] \boldsymbol{x}=\boldsymbol{x}^{T} \boldsymbol{x} .
$$

By the fact $\boldsymbol{x}^{T}\left[\boldsymbol{B}^{T}(k) \boldsymbol{B}(k)\right] \boldsymbol{x} \leq \boldsymbol{x}^{T} \boldsymbol{x}$, the equality leads to

$$
\boldsymbol{x}^{T}\left[\boldsymbol{B}^{T}(k) \boldsymbol{B}(k)\right] \boldsymbol{x}=\boldsymbol{x}^{T} \boldsymbol{x}
$$

with probability one. On the other hand, by the link failure assumption, there exists a set $A_{1} \subset \Omega$ such that $P\left(A_{1}\right)>0$, and for each $\omega \in A_{1}$, the associated matrix value $\boldsymbol{B}(k)=\boldsymbol{I}-$ $\epsilon \boldsymbol{L}$. Without loss of generality, we can assume that $A_{1}$ has been chosen in such a manner that, for any $\omega \in A_{1}$, (14) also holds.

By noticing the fact that, for any $z \in \mathbb{R}^{n}$

$$
\boldsymbol{z}^{T}\left[\boldsymbol{B}^{T}(k) \boldsymbol{B}(k)\right] \boldsymbol{z} \leq \boldsymbol{z}^{T}(\boldsymbol{I}-\epsilon \boldsymbol{L})^{2} \boldsymbol{z} \leq \boldsymbol{z}^{T} \boldsymbol{z}
$$

we obtain from (14)

$$
\boldsymbol{x}^{T}(\boldsymbol{I}-\epsilon \boldsymbol{L})^{2} \boldsymbol{x}=\boldsymbol{x}^{T} \boldsymbol{x} .
$$

Hence, (16) implies that $\boldsymbol{x}$ is the eigenvector of $\boldsymbol{I}-\epsilon \boldsymbol{L}$ associated with the eigenvalue 1 , which further implies that $\boldsymbol{x} \in \operatorname{span}\left\{\mathbf{1}_{n}\right\}$. Denote $\boldsymbol{x}=c \mathbf{1}_{n}$, where $c$ is a constant. By substituting $\boldsymbol{x}=c \mathbf{1}_{n}$ into the left-hand side of (14), we obtain $\boldsymbol{x}^{T}\left[\boldsymbol{B}^{T}(k) \boldsymbol{B}(k)\right] \boldsymbol{x}=0$ for each $\omega \in \Omega$, which contradicts with (14) and the fact that $\boldsymbol{x} \neq 0$. Hence, we conclude that the largest eigenvalue $\rho$ of $E\left[\boldsymbol{B}^{T}(k) \boldsymbol{B}(k)\right]$ is in the interval $[0,1)$.

Finally, by elementary calculation, we obtain the convergence rate estimate

$$
E\left|\boldsymbol{x}(k)-\operatorname{Ave}(\boldsymbol{x}(0)) \mathbf{1}_{n}\right|^{2} \leq \rho^{k} E\left|\boldsymbol{x}(0)-\operatorname{Ave}(\boldsymbol{x}(0)) \mathbf{1}_{n}\right|^{2} .
$$

In fact, we have the simplified expression

$$
\begin{aligned}
\boldsymbol{B}^{T}(k) \boldsymbol{B}(k) & =[\boldsymbol{I}-\epsilon \boldsymbol{L}(k)]\left[\boldsymbol{I}-(1 / n) \mathbf{1}_{n} \mathbf{1}_{n}^{T}\right]^{2}[\boldsymbol{I}-\epsilon \boldsymbol{L}(k)] \\
& =[\boldsymbol{I}-\epsilon \boldsymbol{L}(k)]\left[\boldsymbol{I}-(1 / n) \mathbf{1}_{n} \mathbf{1}_{n}^{T}\right][\boldsymbol{I}-\epsilon \boldsymbol{L}(k)] \\
& =[\boldsymbol{I}-\epsilon \boldsymbol{L}(k)]^{2}-(1 / n) \mathbf{1}_{n} \mathbf{1}_{n}^{T}
\end{aligned}
$$

and therefore, $\rho$ is also given as the largest eigenvalue of the positive semidefinite matrix $E[\boldsymbol{I}-\epsilon \boldsymbol{L}(k)]^{2}-(1 / n) \mathbf{1}_{n} \mathbf{1}_{n}^{T}$.

\section{Simulation Results and Discussions}

In this section, we first introduce the simulation setup. Then, some simulation results are presented and discussed to show the performance of the proposed scheme.

\section{A. Simulation Setup}

In the simulations, we assume that all secondary users are experiencing independent identically distributed Rayleigh fading without spatial correlation. Each secondary user uses an energy detector. We directly simulate the output $Y$ of the energy detector in our simulations. When the primary user is absent, $Y$ is a random quantity with chi-square distribution. When the primary user is present, $Y$ may be denoted as the sum of two independent random variables [24], [25]. The parameters of $Y$ depend on the average SNR in the Rayleigh fading [see (2) and (3)]. The simulations are done under three test conditions. In the first condition, every user has the same average $\operatorname{SNR}(\bar{\gamma})$, which is $10 \mathrm{~dB}$. In the second condition, each user has a different average $\operatorname{SNR}(\bar{\gamma})$ varying from 5 to $9 \mathrm{~dB}$. In the third condition, each user a has different average $\operatorname{SNR}(\bar{\gamma})$ varying from 5 to $15 \mathrm{~dB}$. The relevant information of primary users, such as the position, the moving direction, and the moving velocity, is unknown to the secondary users.

We compare the performance of the proposed scheme with that of an existing OR-rule cooperative-sensing scheme [7], [11], [12], which is better than the AND-rule and MAJORITYrule in many cases of practical interest [7], [12]. In the OR-rule cooperative-sensing scheme, each secondary user makes a local spectrum-sensing decision, which is a binary variable: A "one" denotes the presence of a primary user, and a "zero" denotes its absence. Then, all of the local decisions are sent to a data collector to sum up all local decision values. If the sum is greater than or equal to one, a primary user is believed to be present.

In the first stage of spectrum sensing, after time synchronization, every secondary user performs energy detection with $T W=5$ individually to get local measurement $Y_{i}$ at the selected center frequency $f_{s}$ and the bandwidth of interest $W$. To set up the initial energy vector $\boldsymbol{X}(0)$, we set $x_{i}(0)=Y_{i}$.

In the second stage, the existing method and the proposed consensus algorithm (6) are conducted based on fixed graph models, whereas the proposed consensus algorithm (12) is run based on random graph models. For fixed graphs, the basic requirement is to set up duplex wireless channels. In the simulations, we consider a network topology with ten secondary users that establish a graph $G=\{\mathcal{N}, \mathcal{E}\}$, as shown in Fig. 2(a). For random graphs, we use the same set of nodes as in Fig. 2(b) but replace solid lines with dotted lines, which have probabilities of link failure of $40 \%$ [refer to Fig. 2(b)]. The links in those figures stand for bidirectional wireless links. With regard to link-failure probabilities, they mean both directions will fail to work in case of link failure. We also consider a network topology with 50 nodes in the simulations, which is shown in Fig. 3. All of the 50 nodes are randomly located. The links in the 50-node network have probabilities of failure of $40 \%$.

\section{B. Convergence of the Consensus Algorithm}

Fig. 4(a) and (b) shows the estimated primary user energy in the network with a ten-node fixed graph. We can observe that, although the initially sensed energy greatly varies due to their different wireless channel conditions for different secondary nodes, a consensus will be reached after several iterations. The step sizes $\epsilon$ have effects on the convergence rate of the consensus algorithm. According to (7) and (12), a value should be selected for $\epsilon$ such that $0<\epsilon<\Delta^{-1}$. Since the maximum number of neighbors of a node in Fig. 2(a) and (b) is 5, $\Delta=5$. Then, $0<\epsilon<0.2$.

Here, we provide some discussion about the choice of the parameter $\epsilon$. First, given the network topology, we may construct the associated Laplacian $\boldsymbol{L}$ as a $10 \times 10$ matrix. Because of 


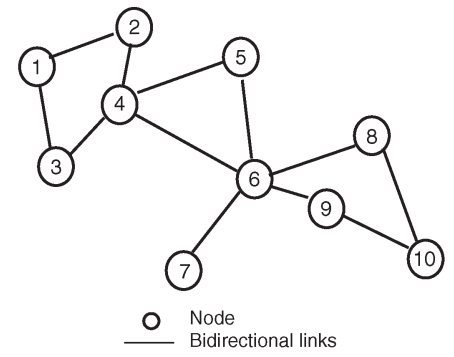

(a)

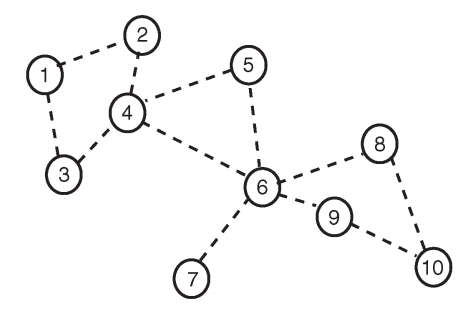

- Node

(b)

Fig. 2. Network topology with ten nodes in the simulations. (a) Fixed graph. (b) Random graph.

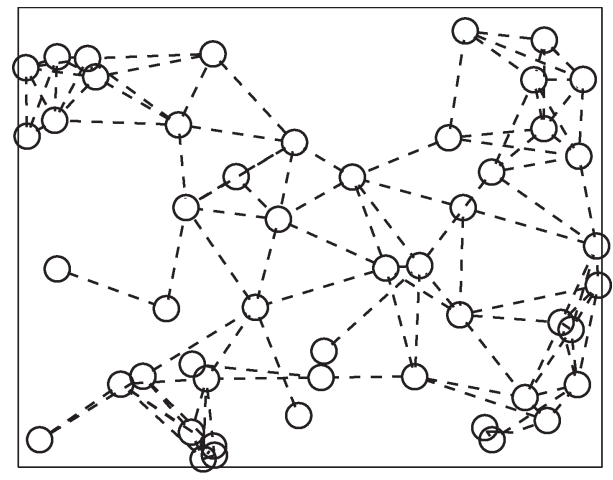

Fig. 3. Network topology with 50 nodes in the simulations.

space limitations, $\boldsymbol{L}$ is not displayed. The eigenvalues of $\boldsymbol{L}$ are listed as follows:

$$
\begin{array}{ccccc}
0 & 0.3416 & 0.8400 & 1.4239 & 2.0000 \\
2.0000 & 3.0000 & 3.1373 & 4.9411 & 6.3161 .
\end{array}
$$

On the interval $(0,0.2)$, the spectral gap $g(\epsilon)$ may be shown to be

$$
g(\epsilon)=1-0.3416 \epsilon
$$

which monotonically decreases on $(0,0.2)$. We note that, for this specific network topology, when $\epsilon=0.2$, the resulting matrix $\boldsymbol{P}=I-\epsilon \boldsymbol{L}$ is ergodic. On the interval $(0,0.2]$, the spectral gap is maximized at $\epsilon=0.2$.

Here, we select two values for $\epsilon$, i.e., 0.1 and 0.19 , in Fig. 4(a) and (b), respectively. We can see that the algorithm converges faster when $\epsilon=0.19$ than that when $\epsilon=0.1$, which is due to the fact that $\epsilon=0.19$ corresponds to a larger spectral gap $g(0.19)$. After about five iterations in Fig. 4(b), the difference between the nodes is less than $1 \mathrm{~dB}$, which indicates that a consensus has been achieved. Fig. 5 shows the estimated primary user energy in the network with a random graph when $\epsilon=0.19$.

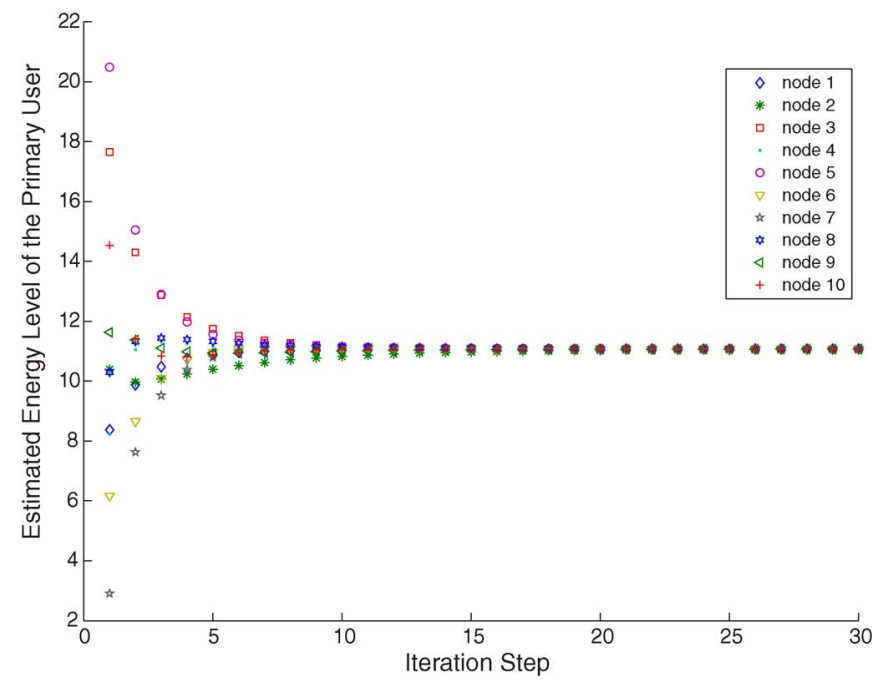

(a)

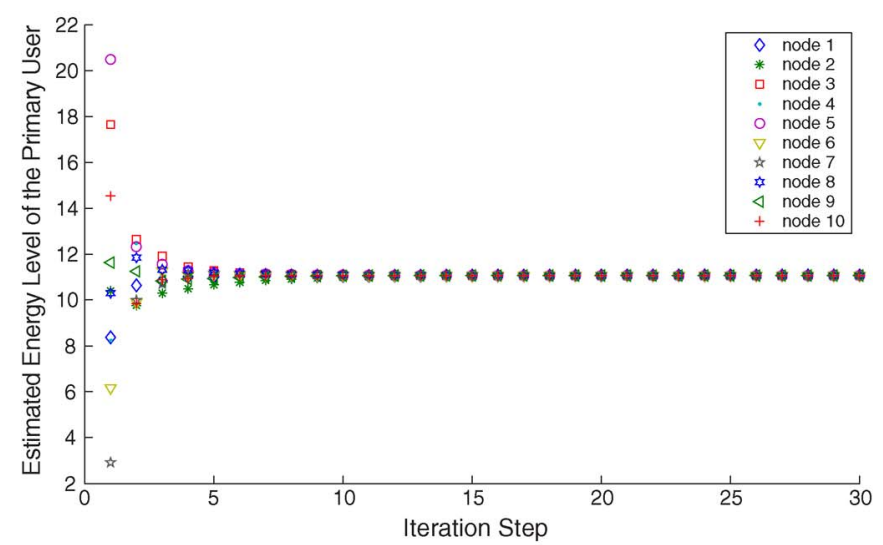

(b)

Fig. 4. Convergence of the network with a ten-node fixed graph. (a) Fixed graph $(\epsilon=0.1)$. (b) Fixed graph $(\epsilon=0.19)$.

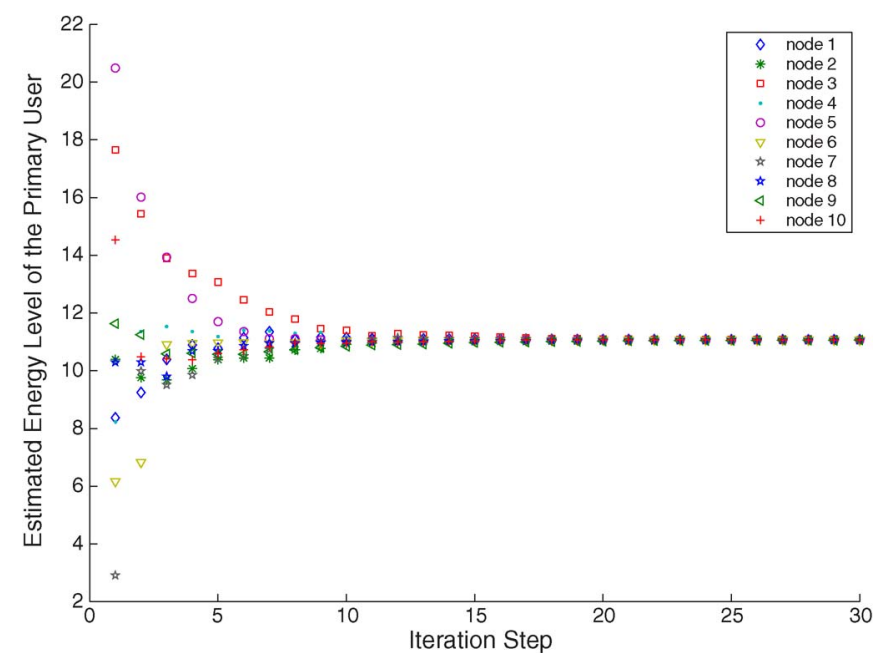

Fig. 5. Convergence of the network with a ten-node random graph $(\epsilon=$ $0.19)$.

Comparing Fig. 5 with Fig. 4(b), we can see that the algorithm more slowly converges in the random graph case due to the random link failure in the CR network. In Fig. 5, after about ten iterations, the difference between the nodes is less than $1 \mathrm{~dB}$, which indicates that a consensus has been achieved. 


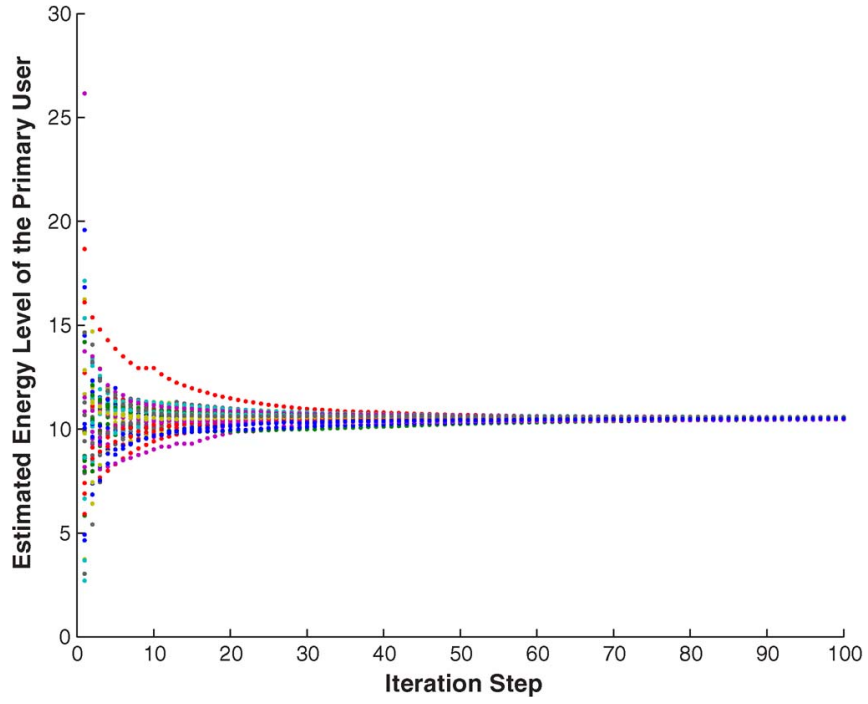

Fig. 6. Convergence of the network with a 50-node random graph $(\epsilon=0.15)$.

Fig. 6 shows the convergence performance for the 50-node network. $\epsilon=0.15$ is used. We can observe that the algorithm more slowly converges in the 50-node network, compared with the ten-node network, due to a larger number of nodes. Nevertheless, after about 30 iterations, the difference between the nodes is less than $1 \mathrm{~dB}$, which indicates that a consensus has been achieved.

\section{Scenario One}

We compare the performance of the proposed scheme with that of an existing OR-rule cooperative sensing scheme [7], [11], [12]. Before the comparison, let us briefly discuss the relationship between $P_{m}$ (probability of missing detection) $=$ $1-P_{d}$ (probability of detection) and $P_{f}$ (probability of false alarm). The fundamental tradeoff between $P_{m}$ and $P_{f}$ has different implications in the context of spectrum sensing [3]. A high $P_{m}$ will result in the missing detection of primary users with high probability, which, in turn, increases the interference to primary users. On the other hand, a high $P_{f}$ will result in low spectrum utilization since false alarms increase the number of missed opportunities (white spaces). As expected, $P_{f}$ is independent of $\gamma$ since, under $H_{0}$, there is no primary signal.

Figs. 7 and 8 show $P_{f}$ versus $P_{m}$. We can see that the proposed algorithm has better performance than the existing OR-rule cooperative sensing scheme. The numbers beside the curves are the corresponding thresholds $\lambda$ in decibels. In Fig. 7, where each secondary user has the same average SNR of $10 \mathrm{~dB}$, if the threshold $\lambda$ is in the range of 11.4-12 dB, both $P_{f}$ and $P_{m}$ can simultaneously drop below the probability of $10^{-2}$ for the proposed consensus algorithm in both fixed and random graphs. In addition, the results are the same between the fixed and random models. In comparison, to reach the same goal, the existing OR-rule method must set $\lambda$ to be about $14.8 \mathrm{~dB}$, which has far worse $P_{m}\left(10^{-2}\right.$ versus $\left.10^{-3}\right)$ with regard to the same $P_{f}$ level $\left(10^{-2}\right)$.

In condition two, secondary users undergo different average SNRs varying from 5 to $9 \mathrm{~dB}$. In condition three, secondary users undergo different average SNRs varying from 5 to $15 \mathrm{~dB}$.

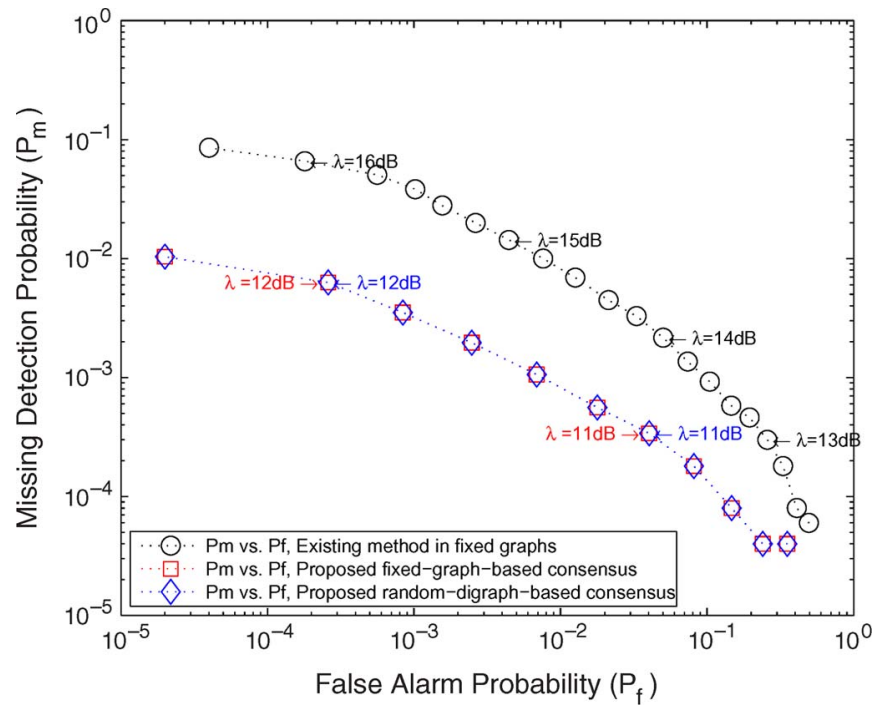

Fig. 7. Missing detection probability $P_{m}$ versus false alarm probability $P_{f}$. (Each secondary user has the same average SNR, i.e., $\bar{\gamma}=10 \mathrm{~dB}$.)

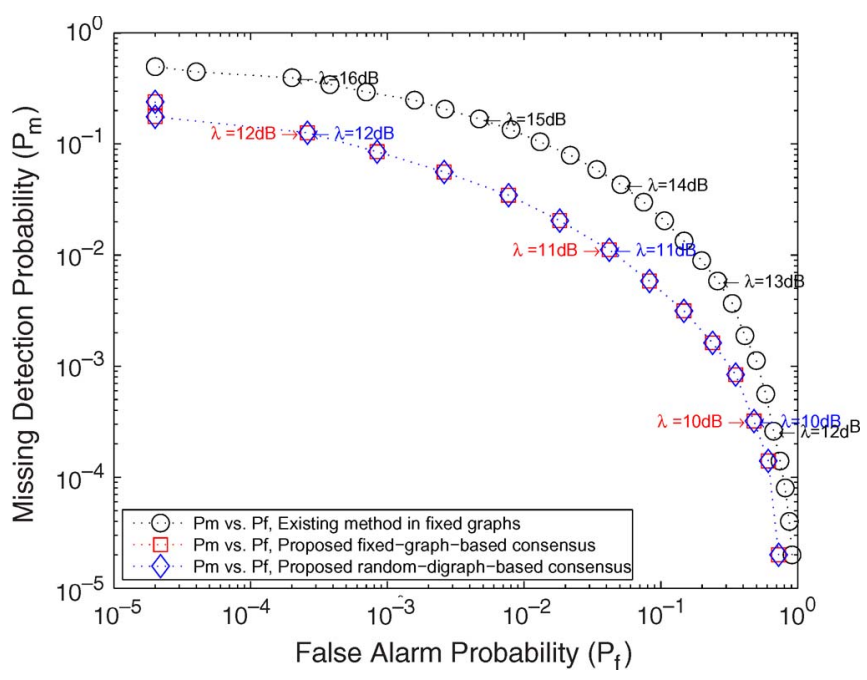

Fig. 8. Missing detection probability $P_{m}$ versus false alarm probability $P_{f}$. (Each secondary user has different average SNRs varying from 5 to $9 \mathrm{~dB}$.)

Similar results are demonstrated in Figs. 8 and 9 for conditions two and three, respectively.

\section{Scenario Two}

Next, we examine the performance of detection probabilities $P_{d}$ to find out the sensitivity in detecting the primary user's presence. Fig. 10 shows $P_{d}$ (detection probability $=1-P_{m}$ ) versus the average SNR $\bar{\gamma}$ of secondary users. Condition one is used in this scenario, and the simulation is performed when the average SNR varies from 5 to $10 \mathrm{~dB}$ for all the nodes. The decision threshold $\lambda$ is chosen to keep $P_{f}=10^{-1}$. Timebandwidth product $T W$ is set to be 5, which is the same as before. From Fig. 10, we see that the proposed scheme can have a significant improvement in terms of the required average SNR for detection. In particular, if the probability of detection is expected to be kept above 0.99 (or $P_{m}<10^{-2}$ ), the existing spectrum-sensing scheme requires $\bar{\gamma}=7.8 \mathrm{~dB}$. This required average SNR is higher than that in the proposed consensus scheme, both of which are approximately $6.8 \mathrm{~dB}$. 


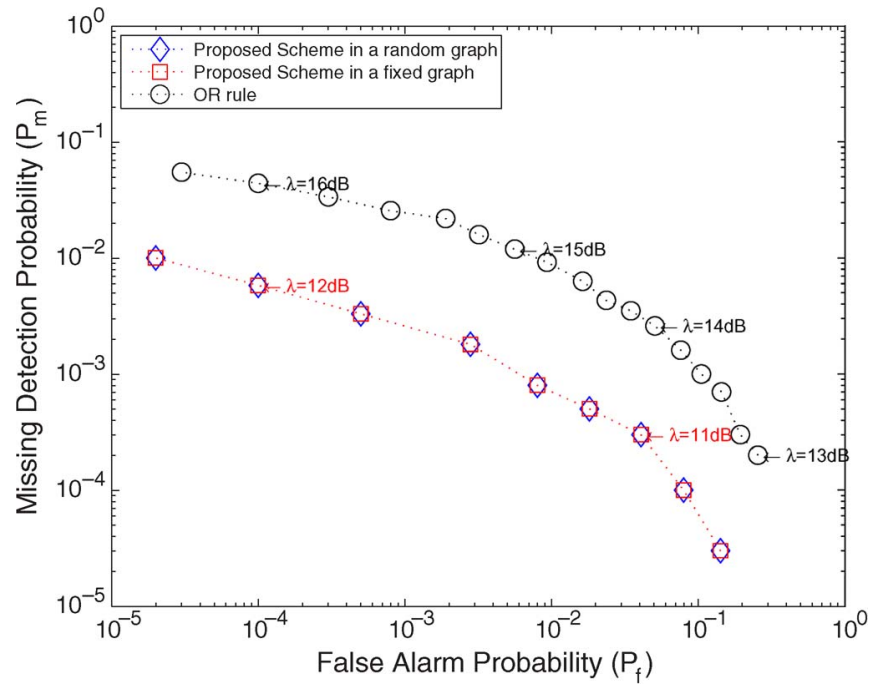

Fig. 9. Missing detection probability $P_{m}$ versus false alarm probability $P_{f}$. (Each secondary user has different average SNRs varying from 5 to $15 \mathrm{~dB}$.)

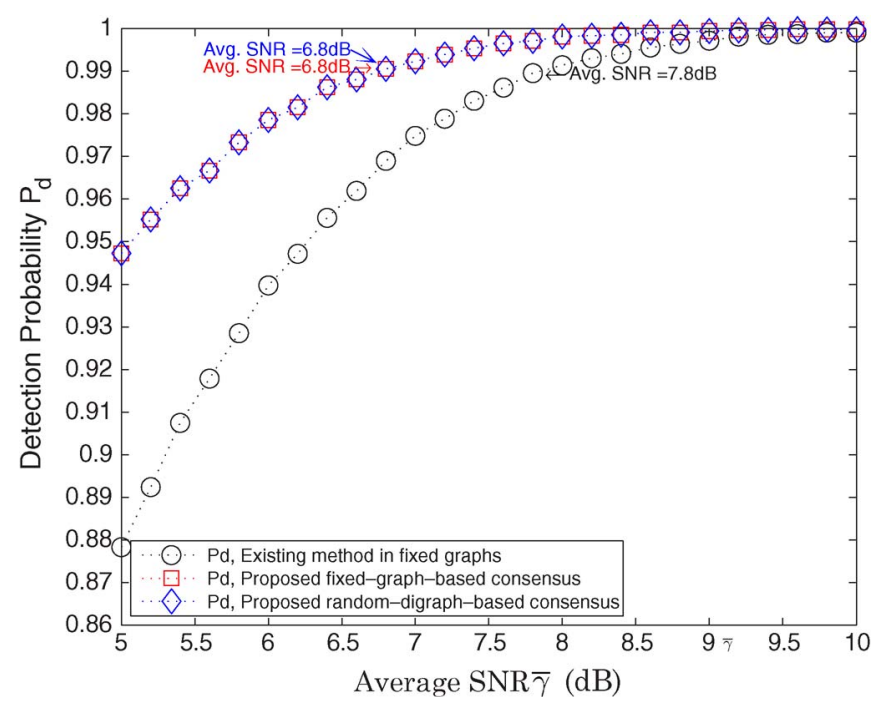

Fig. 10. Simulation results in scenario two. Detection probability $P_{d}$ versus average SNR $\bar{\gamma}\left(P_{f}=10^{-1}, T W=5\right)$.

\section{E. Scenario Three}

In reality, it is unlikely to adjust the threshold $\lambda$ on demand with regard to the different average SNRs. Rather, a fixed threshold that can work in any $\bar{\gamma}$ is much more desirable. We can call it threshold robustness. Therefore, in this scenario, we use condition one and intend to set a predefined threshold $\lambda$ by using (10) to achieve a certain goal. In fact, there are three options when we choose such a goal to keep missing detection probability $P_{m}$ below a certain level, to keep false alarm probability $P_{f}$ around a certain level, or to keep both $P_{m}$ and $P_{f}$ as low as possible.

We first try to keep $P_{m}$ below $10^{-2}$ when all the ten users undergo the same $\bar{\gamma}$ varying from 5 to $10 \mathrm{~dB}$. Fig. 11(a) shows a fixed $\lambda$ that lets $P_{m}$ be below $10^{-2}$ for the average SNR ranging from 5 to $10 \mathrm{~dB}$. As a result, the worst $P_{f}$ decreases from 0.586 by using the existing method to 0.356 in both the random graph and the fixed graph by using the proposed scheme.

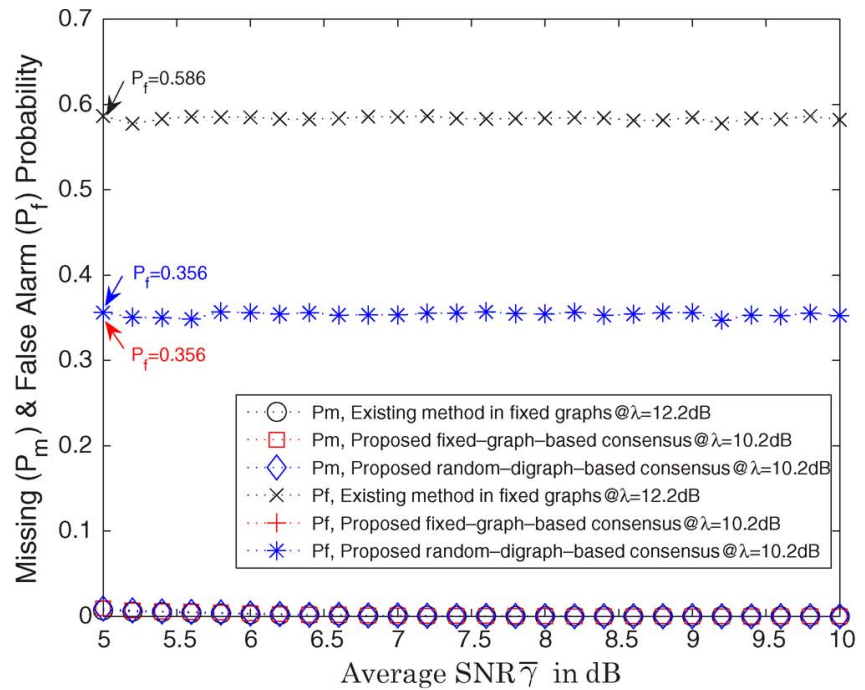

(a)

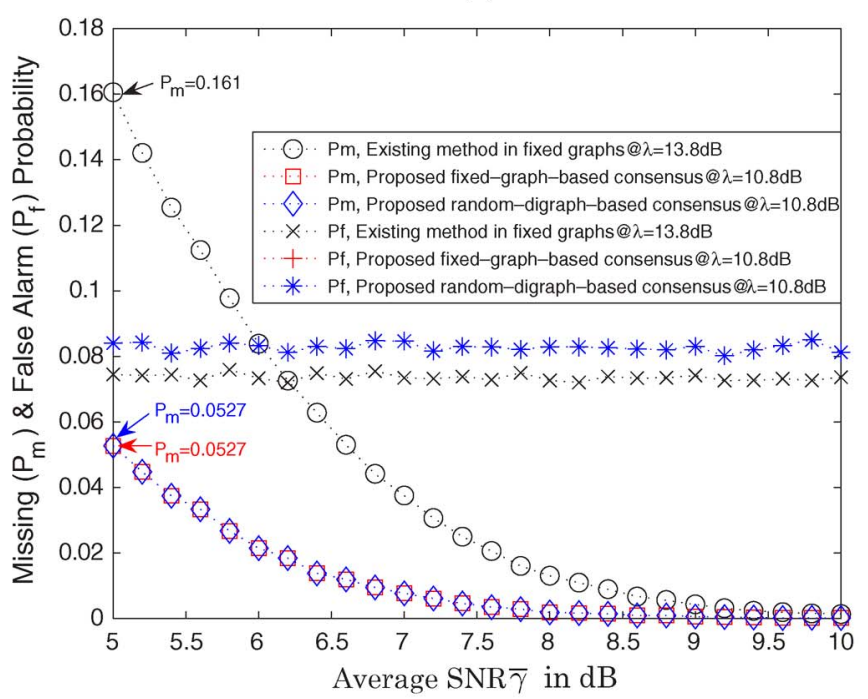

(b)

Fig. 11. Results in simulation scenario three (part one). (a) Missing detection probability $P_{m}$ and false alarm probability $P_{f}$ versus average SNR $\bar{\gamma}$ with fixed threshold $\lambda$ to keep $P_{m}$ below $10^{-2}$, when all the ten users undergo the same $\bar{\gamma}$ varying from 5 to $10 \mathrm{~dB}$. (b) Missing detection probability $P_{m}$ and false alarm probability $P_{f}$ versus average SNR $\bar{\gamma}$ with fixed threshold $\lambda$ to keep $P_{f}$ below $10^{-1}$, when all the ten users undergo the same $\bar{\gamma}$ varying from 5 to $10 \mathrm{~dB}$.

The second option is to let $P_{f}$ always be about $10^{-1}$ when all the ten users undergo $\bar{\gamma}$ varying from 5 to $10 \mathrm{~dB}$. The result is shown in Fig. 11(b), where $P_{f}$ remains about $10^{-1}$. The proposed consensus algorithm performs better in terms of $P_{m}$, decreasing from 0.161 in the existing method to 0.0527 in the proposed method.

In the third option, keep both $P_{m}$ and $P_{f}$ as low as possible. When determining a threshold, we refer to Fig. 12(a), which shows the worst case when all the ten users suffer $\bar{\gamma}=5 \mathrm{~dB}$. For the consensus scheme to have better missing detection performance, the threshold chosen in the proposed scheme should be lower than that in the oR-rule scheme. In Fig. 12(a), we can see that, with the same missing detection probability, the threshold in the proposed scheme is lower than that in the OR-rule scheme. On the other hand, with this lower threshold, a better false alarm probability can be achieved in the 


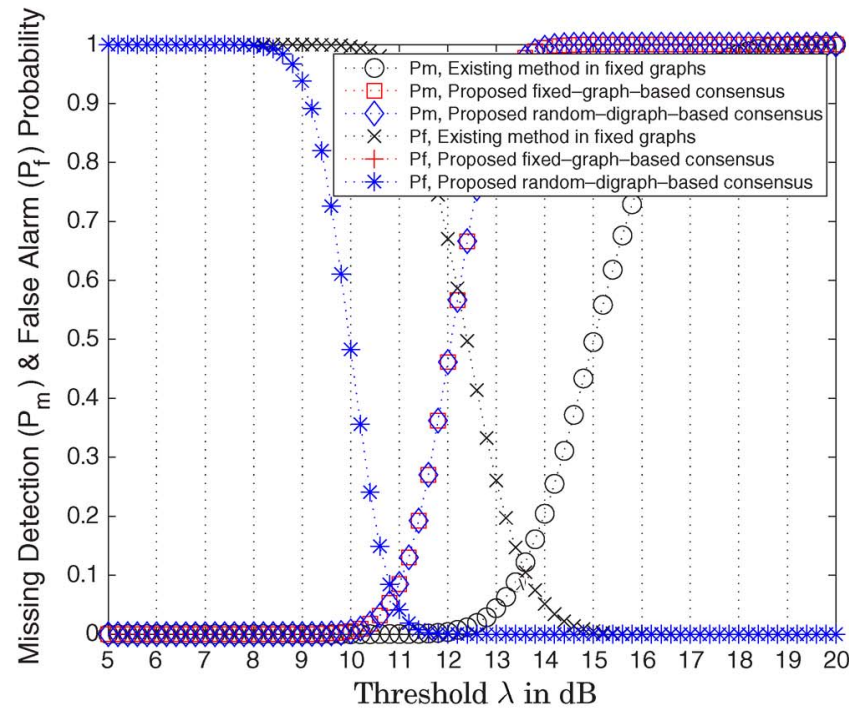

(a)

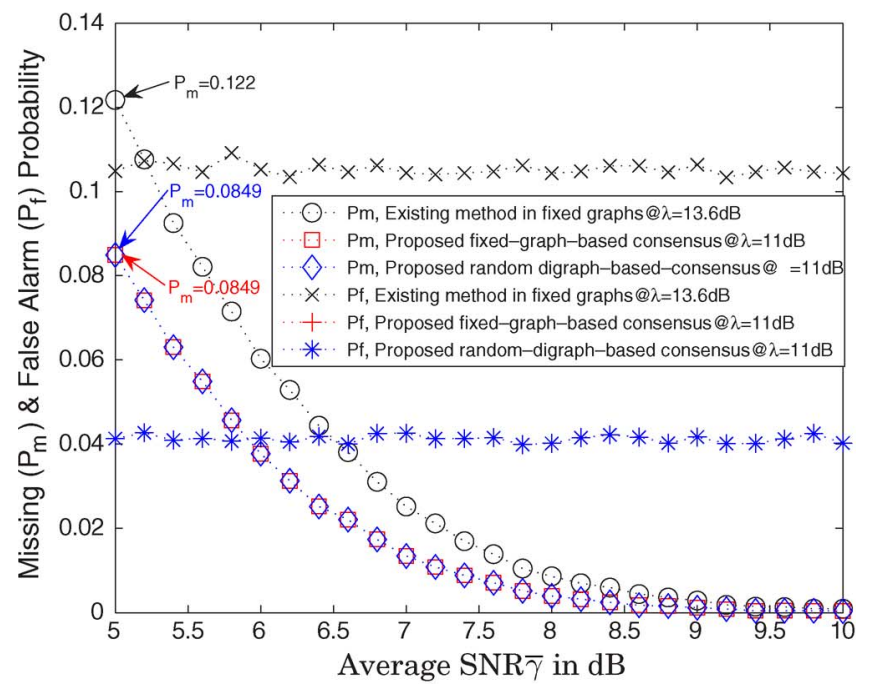

(b)

Fig. 12. Results in simulation scenario three (part two). (a) Missing detection probability $P_{m}$ and false alarm probability $P_{f}$ versus threshold $\lambda$ in decibels with the same $\bar{\gamma}=5 \mathrm{~dB}$ for all users. (b) Missing detection probability $P_{m}$ and false alarm probability $P_{f}$ versus average SNR $\bar{\gamma}$ with fixed threshold $\lambda$ to keep both $P_{m}$ and $P_{f}$ below a certain level, when all the ten users undergo the same $\bar{\gamma}$ varying from 5 to $10 \mathrm{~dB}$.

proposed scheme. The reason is that, when there is no primary user, the output of the energy detector $Y$ of each secondary user is a random quantity with central chi-square distribution [see (2)]. Since $Y$ greatly varies, it is easy for a secondary user to have a false alarm in the oR-rule scheme. By contrast, the consensus scheme does not use the raw data $Y$ to make decisions. Instead, it uses the consensus among the secondary users to make decisions; thus, it can remove some randomness in the raw data $Y$. Therefore, the consensus scheme can have a better false alarm probability than the OR-rule scheme with the same threshold. This can be shown in Fig. 12(a). From Fig. 12(a), we can also observe that both missing detection and false alarm probabilities are low when the threshold is about $11 \mathrm{~dB}$ for the consensus scheme and when the threshold is about 13.6 $\mathrm{dB}$ for the OR-rule scheme. In Fig. 12(a), if we compare the performance of the consensus scheme with a threshold of $11 \mathrm{~dB}$ with that of the oR-rule scheme with a threshold of
13.6 dB, we can see that both missing detection and false alarm probabilities in the consensus scheme are lower than those in the oR-rule scheme. We choose $\lambda=11 \mathrm{~dB}$ for the proposed consensus algorithm and $\lambda=13.6 \mathrm{~dB}$ for the existing method to conduct our numerical studies. Fig. 12(b) shows the result of such a fixed $\lambda$. It is seen that both $P_{m}$ and $P_{f}$ perform better for the proposed algorithm than those of the existing method. $P_{m}$ and $P_{f}$ drop to a relatively low level. This highlights the overall advantage in the so-called threshold robustness for the proposed consensus algorithm. That is, for a given $\lambda$, the proposed consensus algorithm can output less $P_{m}$ and $P_{f}$ than those of the existing method. The algorithm works well in both the fixed and random graphs.

Another observation in scenario three is that when the average SNR rises, $P_{m}$ drops for a given threshold $\lambda$, but $P_{f}$ remains more or less at the same level. This means that, for a fixed $\lambda, P_{m}$ is subject to a change in the average SNR. In contrast, $P_{f}$ is stable, because this parameter deals with the condition of $H_{0}$, where only the collective noises exists.

\section{COnCluding Remarks And Future Work}

In this paper, we have presented a fully distributed and scalable scheme for spectrum sensing based on recent advances in consensus algorithms. Cooperative spectrum sensing is modeled as a multiagent coordination problem. Secondary users can maintain coordination based on only local information exchange without a centralized receiver. Simulation results have been presented to show the effectiveness of the proposed consensus-based scheme. It has been shown that both missing detection probability and false alarm probability can significantly be reduced in the proposed scheme, compared with those in the existing schemes.

In addition, as the real network topologies undergo random changes and the primary user may randomly enter and leave the network, a protocol is necessary to quickly decide when the consensus is considered to be practically reached. If the secondary users cannot efficiently form a decision in finite steps, the energy measurements obtained at the beginning may become obsolete. To address this finite time-detection issue, in implementations, a certain toleration threshold may be used by the users. A secondary user may stop the iteration if it finds that the difference between the states of each neighbor and itself has fallen below the threshold. The choice of threshold depends on empirical studies. Our simulation indicates that the threshold may be chosen to be around a fraction of $1 \mathrm{~dB}$ or close to $1 \mathrm{~dB}$.

One limitation of the proposed scheme is that the choice of the step size $\epsilon$ depends on the maximum number of neighbors of a node in the network. In other words, each node needs to have prior knowledge of an upper bound of the maximum degree of the network. To solve this problem, an alternative approach may be used, which is based on the so-called Metropolis weights [16]. This approach does not need knowledge of the maximum degree of the network. Future work is in progress in this direction. We also want to simplify the data format of detection statistics from each secondary user to save the wireless bandwidth. In addition, as energy detection does not work well for spread-spectrum signals, other approaches will be studied to deal with such networks. 


\section{ACKNOWLEDGMENT}

The authors would like to thank the reviewers for their detailed reviews and constructive comments, which have helped improve the quality of this paper.

\section{REFERENCES}

[1] J. Mitola, "Cognitive radio: An integrated agent architecture for software defined radio," Ph.D. dissertation, Roy. Inst. Technol. (KTH), Stockholm, Sweden, 2000.

[2] S. Haykin, "Cognitive radio: Brain-empowered wireless communications," IEEE J. Sel. Areas Commun., vol. 23, no. 2, pp. 201-220, Feb. 2005

[3] A. Ghasemi and E. Sousa, "Collaborative spectrum sensing for opportunistic access in fading environments," in Proc. IEEE DySPAN, 2005, pp. 131-136.

[4] G. Ganesan and Y. G. Li, "Agility improvement through cooperative diversity in cognitive radio," in Proc. IEEE GLOBECOM, 2005, pp. $2505-2509$.

[5] S. Mishra, A. Sahai, and R. Brodersen, "Cooperative sensing among cognitive radios," in Proc. IEEE ICC, 2006, pp. 1658-1663.

[6] E. Peh and Y.-C. Liang, "Optimization for cooperative sensing in cognitive radio networks," in Proc. IEEE WCNC, 2007, pp. 27-32.

[7] A. Ghasemi and E. S. Sousa, "Opportunistic spectrum access in fading channels through collaborative sensing," J. Commun., vol. 2, no. 2, pp. 71-82, Mar. 2007.

[8] J. Unnikrishnan and V. V. Veeravalli, "Cooperative sensing for primary detection in cognitive radio," IEEE J. Sel. Topics Signal Process., vol. 2, no. 1, pp. 18-27, Feb. 2008.

[9] Z. Quan, S. Cui, and A. H. Sayed, "Optimal linear cooperation for spectrum sensing in cognitive radio networks," IEEE J. Sel. Topics Signal Process., vol. 2, no. 1, pp. 28-40, Feb. 2008.

[10] Y.-C. Liang, Y. Zeng, E. C. Y. Peh, and A. T. Hoang, "Sensing-throughput tradeoff for cognitive radio networks," IEEE Trans. Wireless Commun., vol. 7, no. 4, pp. 1326-1337, Apr. 2008.

[11] R. Chen, J. M. Park, and K. Bian, "Robust distributed spectrum sensing in cognitive radio networks," in Proc. IEEE Infocom, Apr. 2008, pp. 1876-1884.

[12] K. B. Letaief and W. Zhang, "Cooperative communications for cognitive radio networks," Proc. IEEE, vol. 97, no. 5, pp. 878-893, May 2009.

[13] C. Sun, W. Zhang, and K. B. Letaief, "Cluster-based cooperative spectrum sensing in cognitive radio systems," in Proc. IEEE ICC, 2007, pp. 2511-2515.

[14] W. Ren, R. Beard, and E. Atkins, "A survey of consensus problems in multi-agent coordination," in Proc. Amer. Control Conf., 2005, pp. 1859-1864.

[15] R. Olfati-Saber, J. Fax, and R. Murray, "Consensus and cooperation in networked multi-agent systems," Proc. IEEE, vol. 95, no. 1, pp. 215-233, Jan. 2007.

[16] L. Xiao, S. Boyd, and S. Lall, "A scheme for robust distributed sensor fusion based on average consensus," in Proc. 4th Int. Symp. Inf. Process. Sens. Netw., 2005, pp. 63-70.

[17] M. Huang and J. H. Manton, "Stochastic consensus seeking with measurement noise: Convergence and asymptotic normality," in Proc. Amer. Control Conf., 2008, pp. 1337-1342.

[18] W. Irving and J. Tsitsiklis, "Some properties of optimal thresholds in decentralized detection," IEEE Trans. Autom. Control, vol. 39, no. 4, pp. 835-838, Apr. 1994.

[19] J.-F. Chamberland and V. V. Veeravalli, "Wireless sensors in distributed detection applications," IEEE Signal Process. Mag., vol. 24, no. 3, pp. 1625, May 2007.

[20] R. Niu and P. K. Varshney, "Performance analysis of distributed detection in a random sensor field," IEEE Trans. Signal Process., vol. 56, no. 1, pp. 339-349, Jan. 2008

[21] V. Veeravalli, "Decentralized quickest change detection," IEEE Trans. Inf. Theory, vol. 47, no. 4, pp. 1657-1665, May 2001.

[22] D. Cabric, S. Mishra, and R. Brodersen, "Implementation issues in spectrum sensing for cognitive radios," in Proc. 38th Asilomar Conf. Signals, Syst., Comput., 2004, vol. 1, pp. 772-776.

[23] H. Urkowitz, "Energy detection of unknown deterministic signals," Proc. IEEE, vol. 55, no. 4, pp. 523-531, Apr. 1967.

[24] F. Digham, M.-S. Alouini, and M. Simon, "On the energy detection of unknown signals over fading channels," in Proc. IEEE ICC, 2003, vol. 5, pp. 3575-3579.
[25] V. Kostylev, "Energy detection of a signal with random amplitude," in Proc. IEEE ICC, 2002, vol. 3, pp. 1606-1610.

[26] M. Huang and J. H. Manton, "Coordination and consensus of networked agents with noisy measurements: Stochastic algorithms and asymptotic behavior," SIAM J. Control Optim., vol. 48, no. 1, pp. 134-161, Jan. 2009.

[27] C. Godsil and G. Royle, Algebraic Graph Theory. New York: SpringerVerlag, 2001.

[28] E. Seneta, Non-Negative Matrices and Markov Chains. New York: Springer-Verlag, 1981.

[29] L. Elsner, I. Koltracht, and M. Neumann, "On the convergence of asynchronous paracontractions with applications to tomographic reconstruction from incomplete data," Linear Algebra Appl., vol. 130, pp. 65-82, 1990

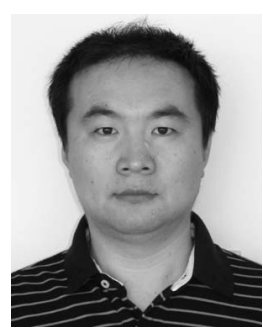

Zhiqiang Li received the B.S. degree in electrical engineering from Qingdao University, Qingdao, China, in 1996 and the M.S. degree in electrical engineering from Tongji University, Shanghai, China, in 1999. From September 2007 to August 2009, he was working toward the M.A.Sc. degree in systems and computer engineering with Carleton University, Ottawa, ON, Canada.

He is currently with the Department of Systems and Computer Engineering, Carleton University. His research interests include cognitive radio, radio resource management, and optimal distributed scheduling algorithms.

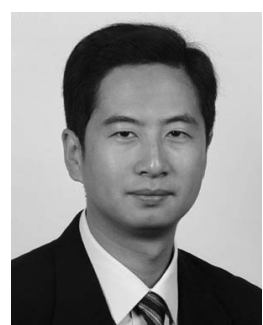

F. Richard Yu (S'00-M'04-SM'08) received the $\mathrm{Ph} . \mathrm{D}$. degree in electrical engineering from the University of British Columbia, Vancouver, BC, Canada, in 2003.

From 2002 to 2004, he was with Ericsson, Lund, Sweden, where he worked on research and development of third genaration cellular networks. From 2005 to 2006, he was with a start-up in California, where he worked on research and development in the areas of advanced wireless communication technologies and new standards. In 2007, he joined the Carleton School of Information Technology and the Department of Systems and Computer Engineering, Carleton University, Ottawa, ON, Canada, where he is currently an Assistant Professor. His research interests include cross-layer design, security, and quality-of-service provisioning in wireless networks.

Dr. Yu has served on the Technical Program Committees (TPCs) of numerous conferences. He was a Co-Chair of the 2009 International Conference on Ultra Modern Telecommunications Workshop on Cognitive Wireless Communications and Networking and a TPC Co-Chair of the 2009 IEEE International Conference on Wireless Communications and Mobile Computing, the Vehicular Technology Conference 2008-Fall Track 4, and the First International Workshop on Wireless Networking for Intelligent Transportation Systems. He was the recipient of the Leadership Opportunity Fund Award from the Canada Foundation of Innovation in 2009 and the Best Paper Awards at the 2009 IEEE/IFIP TrustCom and the 2005 International Conference on Networking.

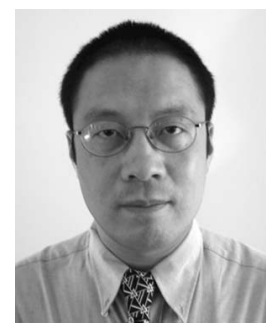

Minyi Huang (S'01-M'04) received the B.Sc. degree from Shandong University, Jinan, China, in 1995, the M.Sc. degree from the Institute of Systems Science, Chinese Academy of Sciences, Beijing, China, in 1998, and the Ph.D. degree from McGill University, Montreal, QC, Canada, in 2003, all in systems and control.

From February 2004 to March 2006, he was a Research Fellow with the Department of Electrical and Electronic Engineering, University of Melbourne, Melbourne, Australia. From April 2006 to June 2007, he was a Research Fellow with the Department of Information Engineering, Research School of Information Sciences and Engineering, Australian National University, Canberra, Australia. In July 2007, he joined Carleton University, Ottawa, ON, Canada, where he is currently an Assistant Professor with the School of Mathematics and Statistics. His research interests include stochastic control and game theory, cooperative multiagent stochastic systems, stochastic algorithms, and wireless networks. 